\title{
Rough Set-Game Theory Information Mining Model Considering Opponents' Information
}

\author{
Ruixia Yan ${ }^{1}$, Liangui Peng ${ }^{2, *}$, Yanxi Xie $^{1}\left(\mathbb{D}\right.$ and Xiaoli Wang ${ }^{1}(\mathbb{D}$ \\ 1 School of Management Studies, Shanghai University of Engineering Science, Shanghai 201620, China; \\ yanruixia@sues.edu.cn (R.Y.); xieyanxi_home@hotmail.com (Y.X.); wxl-0539@163.com (X.W.) \\ 2 School of Business, East China University of Science and Technology, Shanghai 200237, China \\ * Correspondence: y10190176@mail.ecust.edu.cn
}

Citation: Yan, R.; Peng, L.; Xie, Y.; Wang, X. Rough Set-Game Theory Information Mining Model Considering Opponents' Information. Electronics 2022, 11, 244. https:// doi.org/10.3390/electronics11020244

Academic Editor: Maciej Ławryńczuk

Received: 14 October 2021

Accepted: 24 November 2021

Published: 13 January 2022

Publisher's Note: MDPI stays neutral with regard to jurisdictional claims in published maps and institutional affiliations.

Copyright: (c) 2022 by the authors. Licensee MDPI, Basel, Switzerland. This article is an open access article distributed under the terms and conditions of the Creative Commons Attribution (CC BY) license (https:// creativecommons.org/licenses/by/ $4.0 /)$.

\begin{abstract}
In multi-strategy games, the increase in the number of strategies makes it difficult to make a solution. To maintain the competition advantage and obtain maximal profits, one side of the game hopes to predict the opponent's behavior. Building a model to predict an opponent's behavior is helpful. In this paper, we propose a rough set-game theory model (RS-GT) considering uncertain information and the opponent's decision rules. The uncertainty of strategies is obtained based on the rough set method, and an accurate solution is obtained based on game theory from the rough set-game theory model. The players obtain their competitors' decision rules to predict the opponents' behavior by mining the information from repeated games in the past. The players determine their strategy to obtain maximum profits by predicting the opponent's actions, i.e., adopting a first-mover or second-mover strategy to build a favorable situation. The result suggests that the rough set-game theory model helps enterprises avoid unnecessary losses and allows them to obtain greater profits.
\end{abstract}

Keywords: information mining; decision rules; rough set; game theory

\section{Introduction}

The influence of preference on the decision is a research hotspot of rational choice theory. Personal experience and cognition can change the outcome of rational decision making [1]. However, the decision maker can hardly obtain complete and accurate information to support their decisions. The information is often accompanied by noise [2]. Moreover, the decisions also mix subjective judgment inevitably. An uncertain environment is harmful to the healthy development of a company. The player hopes to keep the market advantage by grasping the opponent's actions. Generally, decision makers take measures to collect the opponent's original information. Unfortunately, this information is not always accurate and clear. We need to find a suitable method to mine this information, which includes the opponent's decision rules.

Rough set is a good theory tool to deal with incomplete information [3-6]. Data objects are distinguished and identified by the classification of a large amount of real data. The greatest advantage of this method is based on objective data to obtain the relationships of the objects without any preparatory knowledge and additional information about the data [7,8]. However, rough set can obtain the opponent's decision-making rules, while they cannot give an exact solution. To solve this problem, we should consider the game theory method.

Game theory is one of the core disciplines of decision science. There is an important station in decision analysis [9]. The classical game theory defines the Nash equilibrium and calculates the equilibrium solution. In the standard expression of game theory, the players set is expressed as $O=\left\{o_{1}, o_{2}, \ldots, o_{n}\right\}$, the strategy space of the players is expressed as $S=\left\{s_{1}, s_{2}, \ldots, s_{n}\right\}$, the profit function is expressed as $\Psi=\left\{\psi_{1}, \psi_{2}, \ldots, \psi_{n}\right\}$, and the game theory can be expressed by $G=\{\Theta ; S ; \Psi\}$. In a method system, a non-cooperative game studies how people make decisions to maximize benefits in situations where profits affect each other, that is, the problem of strategy choice. The important difference between a 
cooperative game and non-cooperative game is that the former emphasizes the information exchange within the union and the existence of binding enforceable contracts. The more obvious difference is that the non-cooperative game focuses on the study of individual behavior characteristics, and the cooperative game focuses on the study of collective behavior characteristics. Not only that, but the two games of cooperation and non-cooperation are mutually inclusive and integrated, and they are different manifestations of the same type of things under different conditions, viewed from different angles. There is an inevitable connection between them, as they have common essential characteristics, and they can be unified in an appropriate form. This requires finding out the inner links between competition and cooperation and integrating them. From the perspective of game process and strategy selection, the cooperative game is a special case of the non-cooperative game.

The game method is used to find a balanced solution. However, this approach ignores the preferences of the decision maker. Game theory assumes that the players are completely rational and that each player takes the goal to maximize utility. In many real experiments, decision makers tend to deviate from the results of game theory. It is difficult for people to keep completely rational in the real world. People usually stand in a state of bounded rationality with the characteristic of preferences. Different decision makers have different decision preferences, and different decision preferences may lead to different decision-making rules. It may lead to deviations in results if the differences between decision makers are ignored $[10,11]$ Preferences reflect the type of decision maker. If the opponent's type is uncertain, the decisions will become more difficult. In the existing research, types of uncertainty are only one of the uncertainty game theories, which also include the uncertain payoff function and strategy uncertainty $[12,13]$. In the study of strategic uncertainty, researchers only regard strategies set as indistinguishable sets and use probability methods to divide boundary domains into positive and negative domains [14]. However, this method is not comprehensive. Researchers pay less attention and mine the historical game information.

Based on the existing research, this paper hopes to find the method that can obtain the opponent's decision-making rules. The decision maker determines their strategy based on the opponent's actions. The players obtain the competitors' decision rules by the rough set method from the information of previous, repeated games. The players can predict the opponent's actions by the acquired knowledge so that they can obtain greater profits in the game to maintain market competitive advantages. From the above discussion, rough set can obtain the opponent's decision-making rules, but it cannot give an exact solution. Game theory can provide an equilibrium solution. However, the calculation becomes difficult when the type of players and the strategies are uncertain. To fill the gap, the main contribution of this paper is to build a rough set-game theory (RS-GT) model. It effectively avoids the effects of uncertain information. The rough set mines uncertainty information to obtain the opponents' decision rules, which are used to predict the opponent's action. This method is dependent on objective real data to usefully avoid the interference of subjective factors. Game theory provides an equilibrium solution. Our work can help players to obtain competitive advantages in the market.

In Section 2, the basic models and theoretical knowledge of rough set and game theory are given as strong evidence to support the construction of new algorithm models in this study. In Section 3, we discuss the rough features of complete and incomplete information. Section 4 gives the rough game algorithm model and each calculation process of the algorithm. Section 5 gives the numerical analysis. In Section 6, we discuss the conclusion and future work. Mathematical symbols are presented in Table A1 from Appendix A.

\section{Preliminaries}

In this section, we provide the basic knowledge about rough set and game theory. Rough set is a mathematical tool for characterizing incompleteness and uncertainty. It can effectively analyze various information, such as inaccuracy, inconsistent, and incomplete information. It can also analyze inferential data to discover hidden knowledge. However, rough set theory is still in the development process. There are still some theoretical 
problems to be solved. Combining rough set with other calculation methods to bring out each method's advantage is a worthwhile direction. Game theory tells us what a rational person should do and what the result is. It considers the prediction and the actual behavior of individuals in the game and studies their optimization strategies. However, the heterogeneous characteristics of the decision makers are neglected.

\subsection{Rough Set}

Rough set theory was introduced by Pawlak, in 1982. The development of rough set shows vigorous vitality and affinity. It is widely replied in different fields, i.e., knowledge discovery, data mining, and intelligent decision making [3]. The core of rough set is derived from a pair of lower and upper approximations from the approximate space, based on the equivalence relationship. Therefore, the study of rough set theory focuses on three aspects: equivalence relationship, approximation space, and domain.

Let $U$ be a finite and nonempty set. $E$ is an equivalence relation on $U$. The pair $R=(U, E)$ is called an approximation space [3,4]. The equivalence relation $E$ induces a partition of $U$, denoted by $U / E$. The equivalence class containing $x$ is given by $[x]_{R}=\{y \mid x E y\}$ [15].

The equivalence classes of $E$ are the basic building blocks to construct algebraic rough set approximations. As for a subset $X \subseteq U$, its lower and upper approximations are defined by the following:

$$
\begin{gathered}
\underline{R}(X)=\{x \in U \mid[x] \subseteq X\} \\
\bar{R}(X)=\{x \in U \mid[x] \cap X \neq \varnothing\}
\end{gathered}
$$

$(\underline{R}(X), \bar{R}(X))$, the lower and upper approximations, can be interpreted as a pair of the unary set theory operator $[3,4]$. Let $\underline{R}(X), \bar{R}(X)$ be the lower and upper approximations about $A$. Its approximate accuracy is defined by the following:

$$
d_{R}(X)=\frac{\underline{R}(X)}{\bar{R}(X)}
$$

Let $|X|$ be as the cardinal number of $X, \bar{R}(X) \neq \varnothing$. When $\bar{R}(X)=\varnothing$, it defines $d(A)=1$. The approximate accuracy reflects the degree to which people know set $X$. For $X \subseteq U, 0<d_{R}(X)<1$. The boundary domain is an empty set if $d_{R}(X)=1$ and it is definable. $X$ has a non-empty boundary domain if $d_{R}(X)<1$ and it is undefinable $[16,17]$.

\subsection{Game Theory}

Game theory is a modern branch of intelligent optimization for studying conflicts and cooperation among rational decision makers $[18,19]$. The prisoner's dilemma is a typical example in game theory. There are two prisoners in this game, $O=\left\{o_{1}, o_{2}\right\}$. The police know that the prisoners are guilty, but they lack sufficient evidence. So, the police keep them in separate jails. Every prisoner has two choices, $S=\left\{s_{1}, s_{2}\right\}$, where $s_{1}=$ Confess and $s_{2}=$ Do not Confess. The payoff function for each action is the amount of jail time that a prisoner will receive. These payoffs are expressed in Table 1, called a payoff table.

Table 1. Payoff table for the prisoner's dilemma.

\begin{tabular}{ccc}
\hline & \multicolumn{3}{c}{ Prisoner 2 } \\
\hline Prisoner 1 & Confess & Do Not Confess \\
\hline Confess & $-8,-8$ & $0,-10$ \\
Do not Confess & $-10,0$ & $-2,-2$ \\
\hline
\end{tabular}

From the example, the equilibrium solution is $(-8,-8)$; however, if both prisoners' select do not confess, every prisoner just receives two years. This is the best result. The above example demonstrates one of the explanation strengths of game theory for complex scenarios.

Game theory provides a clear explanation for complex scenarios and can clearly understand the payoff of players and opponents. In a long period of the development 
process, game theory has become a powerful tool for economics, computer science, artificial intelligence, and other disciplines [20]. Game theory is also a practical subject; its basic principles and strategic methods are the behavioral and practical means for modern political and economic activities, financial investment, business decision making, organizational management, and other fields $[21,22]$.

\section{The Features of Complete and Incomplete Information in Rough Set}

Rough set analyzes different types of data information whose classification ability is used to judge the types of information, i.e., complete or incomplete information. In this section, the representation of information in rough set is analyzed in Section 3.1. The representation of complete information and incomplete information in rough set is analyzed in Section 3.2.

\subsection{The Representation of Information in Rough Set}

In classical game theory, the probability is used to describe the uncertainty of information. However, the probability of a certain player's strategy is hardly obtained. The decisions and judgments often are based on incomplete information or knowledge. Compared with probability statistics, evidence theory, fuzzy sets, and other mathematical tools, rough set theory has advantages that these theories do not have. One of the main advantages of rough set theory is that it does not require any preliminary or additional information about the data. Therefore, rough set provides theoretical support for people to study incomplete information and its characteristics. Rough set theory is considered a new mathematical tool for dealing with inaccurate, uncertain, and fuzzy knowledge, and it does not use probabilistic methods to describe the uncertainty of data. Rough set discovers knowledge and rules by the classification ability from real data.

This paper discusses the player's behavior of the strategy adopted in the market. The probability is obtained by statistical data or probability distribution function, that is, the probability is obtained from the proportion that has adopted this strategy in the past. to judge what strategy an enterprise adopts in the market competition. Rough set identifies the firm's strategy based on the real information of the firm.

There is a two-dimensional table to illustrate it, as shown in Table 2. To obtain the frequency of what strategy the company adopts, we need to know the proportion of this strategy in the $S$ column from Table 2 . The number of times which the enterprise adopts the strategy $i$ is $v_{i}$. The frequency value is $v_{i} / n$ which is the firm's adoption probability of strategy $i$. This judgment method is random and unreliable. There is a great reference risk for players.

Table 2. Strategy information.

\begin{tabular}{|c|c|c|c|c|c|c|}
\hline$U$ & $X_{1}$ & $X_{2}$ & $X_{3}$ & $\ldots$ & $X_{\mathrm{n}}$ & $S$ \\
\hline 1 & $\mathrm{a}_{11}$ & $\mathrm{a}_{12}$ & $\mathrm{a}_{13}$ & $\ldots$ & $\mathrm{a}_{1 \mathrm{n}}$ & $\mathrm{s}_{1}$ \\
\hline 2 & $\mathrm{a}_{21}$ & $a_{22}$ & $\mathrm{a}_{23}$ & $\ldots$ & $\mathrm{a}_{2 \mathrm{n}}$ & $\mathrm{s}_{2}$ \\
\hline 3 & $a_{31}$ & $\mathrm{a}_{32}$ & $\mathrm{a}_{33}$ & $\ldots$ & $\mathrm{a}_{3 \mathrm{n}}$ & $\mathrm{s}_{3}$ \\
\hline$\ldots$ & $\ldots$ & $\ldots$ & $\ldots$ & $\ldots$ & $\ldots$ & $\ldots$ \\
\hline $\mathrm{N}$ & $a_{n 1}$ & $a_{n 2}$ & $a_{n 3}$ & $\ldots$ & $a_{n n}$ & $\mathrm{~S}_{\mathrm{n}}$ \\
\hline
\end{tabular}

To discuss this case, the row information is classified by rough set to abtain the rules. We assume that the information set can determine which strategy the enterprise adopts, that is, the lower approximation of an equivalence class in the information set $X$ is not zero. In the above example, there is an information set that can let enterprise 1 confirm which strategy enterprise 2 will adopt. Otherwise, if there is no element in the information set for the judgment of which strategy enterprise 2 adopts, it happens that a strategy may exist, but the approximate accuracy is 0 . 


\subsection{The Feature Distinction between Complete Information and Incomplete Information in Rough Set}

We assume that the player has accurate knowledge about the characteristics, strategic space, and payoff function of their opponents in a complete information game. The information is complete and certain. Otherwise, it is an incomplete information game. In this section, the completeness of the information is described by rough set.

According to the basic principles of rough set, the uncertainty is caused by the boundary domain. The larger the boundary domain of the set, the lower its accuracy. Therefore, the uncertainty concept of set $X$ is introduced-approximate accuracy.

We assume that enterprise 1 and enterprise 2 are players, and the strategies set of players 2 is $S^{(2)}=\left\{s_{1}^{2}, \ldots, s_{j}^{2}, \ldots, s_{m}^{2}\right\}, j=(1,2, \ldots, m)$. Player 1 adopts a strategy based on the strategies of player 2 in a competitive situation. The information set $X^{(2)}=\left\{x_{1}^{2}, \ldots, x_{g}^{2}, \ldots, x_{l}^{2}\right\}, g=(1,2, \ldots, l)$ of player 2 is the knowledge set of enterprise 1. Player 1 analyzes and forecasts the strategy of player 2 based on the information set, i.e., categorizes the strategies of player 2 . Let $R$ be the equivalence relationship. We assume there are $m$ number of elements in the information set $X^{(2)}$ which can determine what strategy player 2 will take, i.e., $\underline{R}\left(X^{(2)}\right)=\mathrm{m}$. There are $n$ number of elements in the information set $X^{(2)}$, which can judge what strategy enterprise 2 may take, i.e., $\bar{R}\left(X^{(2)}\right)=n$. The approximate accuracy defines the degree to which player 1 is able to determine the strategy that player 2 takes.

$$
d_{R}\left(X^{(2)}\right)=\frac{\left|\underline{R}\left(X^{(2)}\right)\right|}{\left|\bar{R}\left(X^{(2)}\right)\right|}
$$

where $d_{R}\left(X^{(2)}\right)=1$, that is $m=n$. Player 1 can certainly confirm the strategy that player 2 takes. The information is complete, i.e., $\underline{R}\left(X^{(2)}\right)=\bar{R}\left(X^{(2)}\right)$, the boundary domain is empty. When $0<d_{R}\left(X^{(2)}\right)<1$, that is $m \neq n$. Player 1 is not sure of the strategy to be adopted by player 2 , the information is incomplete, i.e., $\underline{R}\left(X^{(2)}\right) \neq \bar{R}\left(X^{(2)}\right)$. The boundary domain is not empty.

We analyze the representation method of complete and incomplete information in rough set theory and give the calculation formula of approximate accuracy. We judge whether the collected information is complete by analyzing approximate accuracy. In the next section, this paper discusses a rough set-game theory model. When the strategy is uncertain, players choose their strategies based on decision rules which are from the mining of the opponents' information.

\section{Rough Set-Game Theory Model in the Situation of Strategic Uncertainty}

The players know all information and strategies about themselves. However, the opponent's information is often incomplete and vague. Because the opponents' strategy is not clear, it will often bring losses or costs for the players. To avoid this situation, the players must know how to obtain the desired knowledge from incomplete information. We propose the model in which the players collect and mine the historical information from repeated games to obtain the opponents' decision rules. The players predict the opponent's actions based on the obtained decision rules. Understanding the opponent's preferences can help the players win the competition. The players hope that the opponents follow their wishes and show that the opponents can obtain a high profit by doing so.

A two-player game includes the following:

Players: $O=\left\{o_{1}, o_{2}\right\}, i=(1,2)$.

Strategies: $S^{(i)}=\left\{s_{1}^{i}, \ldots, s_{j}^{i}, \ldots, s_{m}^{i}\right\}, i=(1,2), j=(1,2, \ldots, m)$.

Payoff Function: $\Psi^{(i)}=\left\{\psi_{1}^{i}, \ldots, \psi_{h^{i}}^{i}, \ldots, \psi_{k}^{i}\right\}, i=(1,2), h=(1,2, \ldots, k) ; \Psi_{h}^{(i)}$ is as payoff function of player $i$. 
When the player chooses the strategy $S_{j}^{(i)}$, the payoff function is given at the same time. As such, the optimal strategy $s_{j}^{(i)^{*}}$ of the player $i$ can be determined by $\psi\left\{s_{-j}^{(i)}, s_{j}^{(-i)}\right\} \leq$ $\psi\left\{s_{-j}^{(i)^{*}}, s_{j}^{(-i)}\right\}, j=(1,2, \ldots, m)$, However, when the opponent has some strategies and the probability cannot be known, the equilibrium solution of the game is hardly calculated. The article assumes that the history of the same game can be observed and the game data based on the history can be used to mine and predict the opponent's behavior. The player relies on their opponents' actions to obtain greater profits. When the opponent's strategy decision is uncertain and the probability is not known, the players' strategy is analyzed and judged according to the collected information [23]. Therefore, the players need to obtain the historical strategy information set of their opponents. The information set is $X^{(i)}=\left\{x_{1}^{i}, \ldots, x_{g}^{i}, \ldots, x_{l}^{i}\right\}, g=(1,2, \ldots, l)$.

The algorithm steps are as Algorithm 1.

Algorithm 1 RS-GT algorithm

Step 1. Collect and organize data. Obtain information tables, $X^{(-i)}$.

Step 2. Check data in the information table. The original data information is classified, the continuous value of the attribute is discretized. Conditional attributes and deci-sion attributes are clarified to obtain a decision table, $D(U / R)$.

Step 3. Reduction set. Firstly, merge objects with indistinguishable relationships and delete redundant information from the decision table. Secondly, to build a distinguishing matrix $\left(H=\left(r_{i} \vee r_{-i}\right) \wedge\left(r_{j} \vee r_{-j}\right)\right.$ and make attributes reduction, $U / R=\left\{[x]_{R}: x \in U\right\}$.

The reduction set $\left(U / R^{\prime}\right)$ is obtained.

Step 4. Let the strategy type be the target condition. According to the reduction set obtained from Step 3, the decision rules are extracted. The result is that the decision rules of opponents are obtained.

Step 5. According to the information from Step 4, the player $i$ obtains the opponent's strategy. Hence, the optimal strategy of player $i$ in the game can be obtained by $\psi\left\{s_{-j}^{(i)}, s_{j}^{(-i)}\right\} \leq \psi\left\{s_{j}^{(i)} * s_{j}^{(-i)}\right\}, i=(1,2), j=(1,2, \ldots, m)$.

Step 6. If the market competition changes, return to Step 1.

The flow chart for the RS-GT algorithm is given, as shown in Figure 1. The players do not know the opponent's strategy. They only know the strategy and related information that the opponent has adopted in the past. Because the information is uncertain and fuzzy, data mining methods are used to obtain the required knowledge. The RS-GT model extracts decision rules by mining historical information. The players predict the opponents' actions and strategies based on the obtained rules to determine their strategies. The players also can develop new market strategies to avoid risks and maximize playoffs. The players obtain the decision knowledge from the mining information of opponents. The players have more understanding about the opponent's preferences to reach the point of "knowing each other". The benefit is obtaining greater profits in the market competition. 


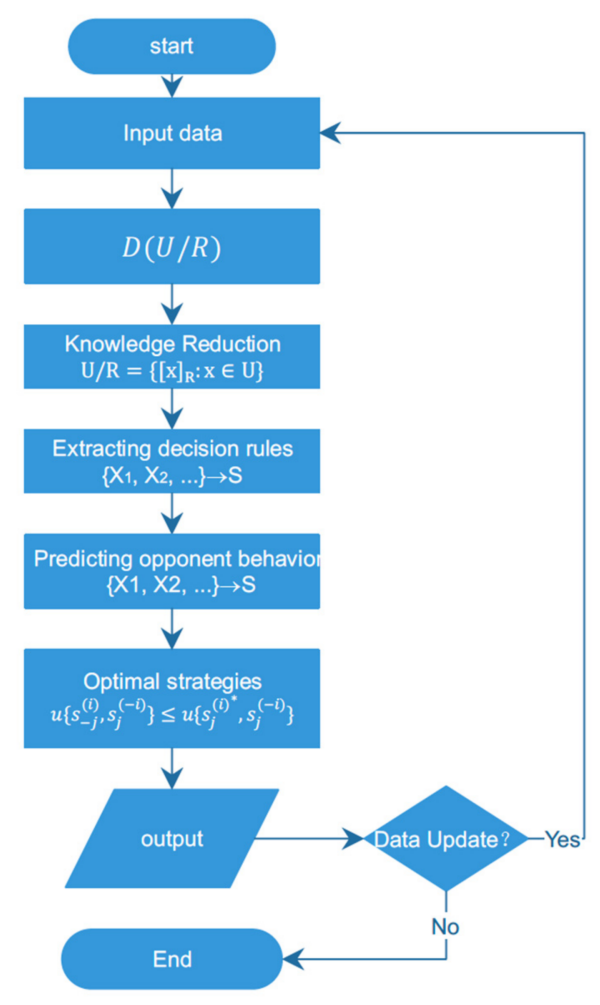

Figure 1. A flow chart for the RS-GT algorithm.

\section{Numerical Analysis}

The RS-GT model is used to solve a competition problem. $B_{1}$ and $B_{2}$ are two peer enterprises in the market. Enterprise $B_{1}$ collects the historical decisions information of $B_{2}$ in the past. Unfortunately, the information is not always clear and certain. Firstly, it assumes that the market environment also is common knowledge for $B_{1}$ and $B_{2}$. $B_{1}$ collects relevant decision information sets of $B_{2}$ from the market, $X^{\left(B_{2}\right)}=\left\{x_{1}, x_{2}, x_{3}, x_{4}, x_{5}, x_{6}\right\}$. In this example, we assume that enterprise $B_{2}$ has two strategies, $S^{\left(B_{2}\right)}=\left\{S_{1}\left(B_{2}\right), S_{2}\left(B_{2}\right)\right\}$. If it can prejudge the market strategy of $B_{2}$, it will be of great significance for enterprise $B_{1}$.

In the example, the strategies of $B_{2}$ are limited. According to the strategies adopted by enterprise $B_{2}$ in the past seven games, $B_{1}$ collects the relevant information at each game. According to the RS-GT algorithm steps Step 1 and Step 2, decision Table 3 is obtained in which high, medium, normal and low are sequentially assigned the values $4,3,2$, and 1 . At the same time, enterprise $B_{2}$ adopts two different strategies under the environment of combined information and takes the numbers 1 and 2 instead.

Table 3. The information table of enterprise $B_{2}$.

\begin{tabular}{cccccccc}
\hline $\boldsymbol{U}$ & $\boldsymbol{X}_{\mathbf{1}}$ & $\boldsymbol{X}_{\mathbf{2}}$ & $\boldsymbol{X}_{\mathbf{3}}$ & $\boldsymbol{X}_{\mathbf{4}}$ & $\boldsymbol{X}_{\mathbf{5}}$ & $\boldsymbol{X}_{\mathbf{6}}$ & $S$ \\
\hline 1 & 2 & 4 & 3 & 4 & 1 & 4 & 1 \\
2 & 4 & 3 & 2 & 4 & 3 & 3 & 1 \\
3 & 3 & 1 & 4 & 1 & 3 & 3 & 2 \\
4 & 2 & 4 & 2 & 3 & 4 & 1 & 2 \\
5 & 4 & 2 & 1 & 1 & 1 & 2 & 1 \\
6 & 2 & 3 & 3 & 2 & 3 & 4 & 2 \\
7 & 2 & 3 & 3 & 2 & 3 & 4 & 1 \\
\hline
\end{tabular}

Step 3. Merging the objects of indistinguishable relationships, the condition attribute values of rows 6 and 7 are the same, but the value of the decision attribute is different. Therefore, the decision table is inconsistent. We delete rows 6 and 7 to obtain the coordination of the decision table, as shown in Table 4. 
Table 4. The reduction information table of enterprise $B_{2}$.

\begin{tabular}{cccccccc}
\hline $\boldsymbol{U}$ & $\boldsymbol{X}_{\mathbf{1}}$ & $\boldsymbol{X}_{\mathbf{2}}$ & $\boldsymbol{X}_{\mathbf{3}}$ & $\boldsymbol{X}_{\mathbf{4}}$ & $\boldsymbol{X}_{\mathbf{5}}$ & $\boldsymbol{X}_{\mathbf{6}}$ & $S$ \\
\hline 1 & 2 & 4 & 3 & 4 & 1 & 4 & 1 \\
2 & 4 & 3 & 2 & 4 & 3 & 3 & 1 \\
3 & 3 & 1 & 4 & 1 & 3 & 3 & 2 \\
4 & 2 & 4 & 2 & 3 & 4 & 1 & 2 \\
5 & 4 & 2 & 1 & 1 & 1 & 2 & 1 \\
\hline
\end{tabular}

We obtain the reduction set by attribute reduction, that is, $\left\{x_{1}, x_{5}\right\},\left\{x_{1}, x_{6}\right\},\left\{x_{2}, x_{5}\right\}$, $\left\{x_{2}, x_{6}\right\},\left\{x_{3}, x_{4}\right\}$.

Step 4. Extract decision rules. We select the reduction set $\left\{x_{1}, x_{5}\right\}$, as shown in Table 5 . $U / S=\left\{\{1,2,5\},\{3,4\}, U / E_{\left(x_{1}, x_{5}\right)}=\{\{1\},\{2\},\{3\},\{4\},\{5\}\}, \operatorname{POS}_{R}(S)=\{1,2,3,4,5\}\right.$, $d_{R}(S)=1$ denotes that the information for the judgment strategy is complete.

Table 5. The reduction information table.

\begin{tabular}{cccc}
\hline $\boldsymbol{U}$ & $\boldsymbol{X}_{\mathbf{1}}$ & $\boldsymbol{X}_{\mathbf{5}}$ & $S$ \\
\hline 1 & 2 & 1 & 1 \\
2 & 4 & 3 & 1 \\
3 & 3 & 3 & 2 \\
4 & 2 & 4 & 2 \\
5 & 4 & 1 & 1 \\
\hline
\end{tabular}

According to the reduction set $\left\{x_{1}, x_{5}\right\}$, we can obtain the decision rules: $\left\{x_{1_{(2)}}, x_{5_{(1)}}\right\} \rightarrow S_{1}\left(B_{2}\right),\left\{x_{1_{(4)}}, x_{5_{(3)}}\right\} \rightarrow S_{1}\left(B_{2}\right),\left\{x_{1_{(3)},} x_{5_{(3)}}\right\} \rightarrow S_{2}{ }^{\left(B_{2}\right)},\left\{x_{1_{(2)}}, x_{5_{(4)}}\right\} \rightarrow S_{2}\left(B_{2}\right)$, $\left\{x_{1_{(4)},} x_{5_{(1)}}\right\} \rightarrow S_{1}\left(B_{2}\right)$.

Step 5. Enterprise $B_{1}$ obtains the opponent's decision rules from Step 4. From $X=\left\{x_{1_{(2)}}, x_{5_{(4)}}\right\}$, enterprise $B_{2}$ takes the strategy $S_{2}{ }^{\left(B_{2}\right)}$. The optimal strategy of enterprise $B_{1}$ can be obtained by $E\left\{S_{j}\left(B_{1}\right), S_{2}\left(B_{2}\right)\right\} \leq E\left\{S_{j}\left(B_{1}\right)^{*}, S_{2}{ }^{\left(B_{2}\right)}\right\}$.

Step 6. When the new information is added to the model, turn to Step 1.

We discuss the decision of $B_{1}$ under a new situation the payoff function is as shown in Table 6. In the above example, the strategy of $B_{2}$ is a prediction result. However, $B_{1}$ hopes to adopt a first-mover strategy to keep an advantage in the competition. The actions of enterprise $B_{2}$ can be affected. The wishes equilibrium of enterprise $B_{1}$ become a reality. For a better explanation, this article assumes that $B_{1}$ can adopt a set of strategies $S^{\left(B_{1}\right)}=\left\{S_{1}\left(B_{1}\right), S_{2}\left(B_{1}\right)\right\} . B_{2}$ can adopt a set of strategies $S^{\left(B_{2}\right)}=\left\{S_{1}\left(B_{2}\right), S_{2}\left(B_{2}\right)\right\}$. The payoff function of both sides is shown as Table 6 [19].

Table 6. Payoff function table.

\begin{tabular}{ccc}
\hline & \multicolumn{3}{c}{$\boldsymbol{B}_{\mathbf{2}}$} \\
\hline $\boldsymbol{B}_{\mathbf{1}}$ & $\boldsymbol{S}_{1}^{\left(\boldsymbol{B}_{2}\right)}$ & $\boldsymbol{S}_{2}^{\left(\boldsymbol{B}_{2}\right)}$ \\
\hline$S_{1}^{B_{1}}$ & 9,9 & 0,8 \\
$S_{2}^{B_{1}}$ & 8,0 & 7,7 \\
\hline
\end{tabular}

In this game, both strategies may be selected in a specific market environment. There are two pure strategy equilibriums, $(9,9)$ and $(7,7)$, in this game. In the above example, it is predicted that the strategy of $B_{2}$ is $S_{2}{ }^{\left(B_{2}\right)}$. In the discussion of classic game theory, the equilibrium point is $(7,7)$. However, the equilibrium is not the expectation of enterprise $B_{1}$. Therefore, $B_{1}$ takes measures to change this equilibrium. Based on the assumptions and results of the RS-GT model, $B_{2}$ chooses strategy $S_{2}{ }^{\left(B_{2}\right)}$ in the next step. However, the 
benefits of $B_{1}$ are worse under strategy $S_{2}{ }^{\left(B_{2}\right)}$. Therefore, $B_{1}$ hopes to change the current situation through the first-mover strategy. In the principle of maximal payoff, it is necessary to give $B_{2}$ sufficient reasons to choose $S_{1}\left(B_{2}\right)$, so that $B_{2}$ has greater benefits. To achieve this goal, $B_{1}$ chooses strategy $S_{1}^{B_{1}}$ through a first-mover strategy and releases the signal to the market. Based on the assumption of maximizing payoff, $B_{2}$ has enough reasons to choose $S_{1}{ }^{\left(B_{2}\right)}$. The equilibrium solution is $(9,9)$. Through the first-mover strategy, $B_{1}$ obtains as much profit as possible in the market competition game. If the information set is $X=\left\{x_{1_{(4)}}, x_{5_{(1)}}\right\}$, and the strategy of $B_{2}$ is $S_{1}\left(B_{2}\right)$, then $B_{1}$ only needs to adopt a second-mover strategy to obtain a higher payoff. In this game, the player's optimal choice is related to the opponent's choice. The player takes action to guide the opponent's actions to maximize the player's payoff.

$B_{1}$ obtains the decision rules of the $B_{2}$ from the mining of collected historical information to predict the strategy of $B_{2}$. $B_{1}$ determines the best action strategy to maximize the payoff. From the analysis, in different environments, decision makers' strategies are different, showing dependence and preference for the environment. As thus, competitors determine whether to adopt a first-mover or second-mover strategy in different situations. In the game above, $B_{1}$ used the first-mover advantage to increase its revenue and avoided the passive situation of adopting the second-mover strategy.

To collect and sort out the information in the historical game and extract the decision rules of competitors, one side of the game can use the acquired knowledge and rules to predict the opponent's actions. From the numerical analysis, it ensures greater gains in the competition and maintains market competitive advantages.

\section{Conclusions}

Decision makers make decisions based on experience. It is often highly uncertain and may lead to misjudgment. As such, players obtain knowledge from historical information to support decision making, hoping to collect the opponents' information as much as possible. However, collecting the information of companies comes with high cost and uncertainty. The information is not always useful. The players use data mining tools to gain knowledge of the opponents' information. Rough set can effectively analyze the fuzzy and uncertain information without any prior information. Rough set theory has an advantage that other probability methods do not have. It is based on a large amount of real data, and has the classification ability to distinguish and identify data objects. Its greatest advantage is the ability, from objective data, to find out the rule between objects. The player uses the obtained decision rules to predict and analyze the opponent's strategy. In the current situation, the question is what kind of strategy should be adopted so that the player can get rid of the disadvantaged situation in the game and obtain the greatest possible payoff.

According to rough set and game theory, this paper finds that rough set can obtain the opponent's decision rules, but they cannot give an exact solution. The game method is used to find a balanced solution, but this approach ignores the preferences of the decision maker. To solve this problem, this paper gives a rough set-game theory model. The player mine and extract the decision rules from the opponents' information by this model. The player uses the obtained decision rules to predict and analyze the opponent's strategy. The player can get rid of the disadvantaged situation in the game and obtain the greatest possible payoff. The players release market signals to guide the decision of their opponents to maintain the market advantage. In the game, strategy $S_{2}\left(B_{2}\right)$ is the opponent's prediction strategy. According to this prediction result, the player adopts the first move to transfer the equilibrium result to $(9,9)$. The player influences the opponent's strategy through its own strategic choices. The method model proposed in the article is a new exploration application of game theory and rough set theory, which combines the two methods' advantages to attain better results.

We discuss an interesting problem. However, this study has other limitations that extensions to this research might address. First, we focus on the two-player game, which raises a question about the generalizability of our findings to multi-player games. Second, 
we focus on the decision rules of the opponents, which raises another question about the generalizability of our findings to different types of opponents; the strategies adopted by enterprises may be very different. In the following research, different types of players are valuable to discuss. It can improve the research of uncertain strategies and the type of players.

Author Contributions: Conceptualization, R.Y. and L.P.; methodology, L.P.; validation, R.Y., L.P., Y.X. and X.W.; formal analysis, R.Y. and L.P.; data curation, Y.X.; writing-original draft preparation, L.P.; writing-review and editing, R.Y.; funding acquisition, X.W. All authors have read and agreed to the published version of the manuscript.

Funding: This research was funded by National Foundation for Philosophy and Social Sciences of China, grant number 19BG234 and 21BGL243; Shanghai Foundation for Philosophy and Social Sciences, grant number 2020BGL005.

Conflicts of Interest: The authors declare no conflict of interest.

\section{Appendix A}

Table A1. Parameters and definition.

\begin{tabular}{cccc}
\hline Parameters & Definition & Parameters & Definition \\
\hline$O$ & The players set & $d_{R}(X)$ & The approximate accuracy \\
$S$ & The strategy space & $X$ & The subset of domain \\
$\Psi$ & The profit function & {$[x]_{R}$} & The equivalence class \\
$U$ & A domain and nonempty set & $\underline{R}(X)$ & Lower approximations \\
$R$ & An approximation space & $\bar{R}(X)$ & Upper approximations \\
$E$ & The equivalence relation & $B_{1}, B_{2}$ & Two enterprises \\
$R, r$ & Attributes set or subset & - & - \\
\hline
\end{tabular}

\section{References}

1. Tversky, A.; Kahneman, D. The framing of decisions and the psychology of choice. Science 1981, 211, 453-458. [CrossRef] [PubMed]

2. Pawlak, Z. Rough sets. Int. J. Comput. Inf. Sci. 1982, 11, 341-356. [CrossRef]

3. Pawlak, Z.; Skowron, A. Rudiments of rough sets. Inf. Sci. 2007, 1, 3-27. [CrossRef]

4. Pawlak, Z. Rough sets, decision algorithms and Bayes' theorem. Eur. J. Oper. Res. 2002, 1, 181-189. [CrossRef]

5. Pawlak, Z.; Wong, S.; Ziarko, W. Rough sets: Probabilistic versus deterministic approach. Int. J. Man-Mach. Stud. 1988, 29, 81-95. [CrossRef]

6. Maldonado, S.; Peters, G.; Weber, R. Credit scoring using three-way decisions with probabilistic rough sets. Inf. Sci. 2020, 507, 700-714. [CrossRef]

7. Yao, Y.Y.; Wong, S. A decision-theoretic framework for approximating concepts. Int. J. Man-Mach. Stud. 1992, 37, 793-809. [CrossRef]

8. Azam, N.; Yao, J. On semantic issues in game-theoretic rough sets. In Proceedings of the Joint IFSA World Congress and NAFIPS Annual Meeting (IFSA/NAFIPS), Edmond, Canada, 24-28 June 2013; pp. 1303-1308.

9. Muthoo, A.; Osborne, M.J.; Rubinstein, A. A Course in Game Theory. Economica 1992, 63, 164. [CrossRef]

10. Jia, X.; Liao, W.; Tang, Z.; Shang, L. Minimum cost attribute reduction in decision-theoretic rough set models. Inf. Sci. 2013, 219, 151-167. [CrossRef]

11. Liu, M.; Ren, H. A New Intuitionistic Fuzzy Entropy and Application in Multi-Attribute Decision Making. Information 2014, 5, 587-601. [CrossRef]

12. Neil, P. A Distance Measure Approach to Exploring the Rough Set Boundary Region for Attribute Reduction. IEEE Trans. Knowl. Data Eng. 2010, 22, 305-317.

13. Chen, Y.; Li, Z.; Zhang, G. Attribute Reduction in an Incomplete Interval-Valued Decision Information System. IEEE Access 2021, 99, 64539-64557. [CrossRef]

14. Peng, L.; Yan, R. Strategies Game Research Based on Rough Sets Attribute Reduction. In Proceedings of the 5th International Conference on Intelligent Computing and Control Systems (ICICCS), Madurai, India, 6-8 May 2021; pp. $1154-1158$.

15. Bashir, Z.; Mahnaz, S.; Malik, M.G.A. Conflict resolution using game theory and rough sets. Int. J. Intell. Syst. 2021, 36, 237-259. [CrossRef]

16. Sang, B.; Guo, Y.; Shi, D.; Xu, W. Decision-theoretic rough set model of multi-source decision systems. Int. J. Mach. Learn. Cybern. 2018, 9, 1941-1954. [CrossRef] 
17. Sharma, H.K.; Kumari, K.; Kar, S. A rough set approach for forecasting models. Decis. Mak. Appl. Manag. Eng. 2020, 3, 1-21. [CrossRef]

18. Herbert, J.P.; Yao, J.T. Game-Theoretic Rough Sets. Fundam. Inform. 2011, 108, 267-281.

19. Abdalzaher, M.S.; Seddik, K.; Elsabrouty, M.; Muta, O.; Furukawa, H.; Abdel-Rahman, A.B. Game Theory Meets Wireless Sensor Networks Security Requirements and Threats Mitigation: A Survey. Sensors 2016, 16, 1003. [CrossRef] [PubMed]

20. Myerson, R.B. Game Theory: Analysis of Conflict; Harvard University Press: Cambridge, MA, USA, 1991.

21. Askari, G.; Gordji, M.E.; Park, C. The behavioral model and game theory. Palgrave Commun. 2019, 5, 56-61. [CrossRef]

22. Abdalzaher, M.S.; Seddik, K.; Muta, O. An effective Stackelberg game for high-assurance of data trustworthiness in WSNs. In Proceedings of the IEEE Symposium on Computers and Communications (ISCC), Heraklion, Greece, 3-6 July 2017; pp. 1257-1262. [CrossRef]

23. Fudenberg, D.; Tirole, J. Game Theory. Economica 1992, 60, 841-846. 